\title{
Structure Formation Dynamics in Drawing Silica Photonic Crystal Fibres
}

\author{
Wenyu WANG ${ }^{1 *}$, Ghazal TAFTI ${ }^{1}$, Mingjie DING ${ }^{1}$, Yanhua LUO ${ }^{1}$, Yuan TIAN $^{1}$, Shuai \\ WANG $^{1,2}$, Tomasz KARPISZ ${ }^{3}$, John CANNING ${ }^{1,4}$, Kevin COOK ${ }^{1,4}$, Gang-Ding PENG ${ }^{1 *}$ \\ ${ }^{I}$ National Fibre Facility, Photonics \& Optical Communication, School of Electrical Engineering and \\ Telecommunications, University of New South Wales, Kensington, NSW 2052, Australia \\ ${ }^{2}$ Henan Key Laboratory of Laser and Opto-Electric Information Technology, School of Information Engineering, \\ Zhengzhou University, Henan 450052, People's Republic of China \\ ${ }^{3}$ Warsaw University of Technology, 26-600, Radom, Poland \\ ${ }^{4}$ interdisciplinary Photonics Laboratories (iPL), School of Electrical \& Data Engineering, UTS and School of \\ Chemistry, The University of Sydney, NSW 2007 \& 2006 Australia
}

\begin{abstract}
The special features of photonic crystal fibres (PCFs) are achieved by their air hole structures. PCF structure is determined and formed by its origin preform design and drawing process. Therefore, structure formation dynamics in drawing PCF is important for the fabrication of PCF achieving desirable structure and thus the intended feature. This paper will investigate structure formation dynamics of PCF drawing in relation to key parameters and conditions, such as hole dimension, temperature, pressure, etc.
\end{abstract}

Keywords: Photonic crystal fibre (PCF), structure formation, hole dimension, hole position, hole shift.

\section{Introduction}

Photonic crystal fibres (PCFs) have attracted lots of interest as they could provide special features that conventional fibres cannot achieve [1], such as near-endlessly single mode [2], strong optical nonlinear effects [3] and high-birefringence [4]. Therefore, they are widely used in (but not limited to) fibre lasers [5-7], fibre sensors $[8,9]$, and nonlinear devices $[3,10]$.

PCF features are mainly determined by the geometry structure of the air holes such as hole dimension, hole shape and hole position [2]. However, hole deformation may happen in the drawing process due to the viscosity and surface tension of the material. Therefore, it is essential to understand the structure formation dynamics in drawing PCF to achieve the original hole structure in the preform by accurate controlling drawing conditions like furnace temperature $T_{d}$, drawing pressure $P_{d}$, feeding rate $V_{f}$, and drawing rate $V_{d}$.

To understand the structure formation in drawing process, much theoretical analysis work has been done. The drawing process modeling of PCF [11,12] related to hole collapse was setup in [13]. Fitt et al [14, 15] carried out the modelling of a single capillary drawing process under isothermal condition, and the stability of drawing capillaries has been reported in [16, 17]. The control of hole expansion was theoretically studied [18] and the method of predicting hole dimension disregarding viscosity was provided [19]. Furthermore, in the drawing process, the transverse radiative heat transfer inside the silica preform was numerically analyzed [12, 20, 21]. In addition to these theoretical studies, several experiments were carried out to explore proper PCF drawing parameters. For example, the relationships of the hole dimension and the spacing pitch between pressure[22], temperature, feeding rate, feeding time, and capillary wall thickness were reported [23-26]. However, directly taking advantage of these results is not practical due to the structural and design differences in drawing facilities. Thus, in order to customize the PCF drawing in our own draw tower, the relationship between the structure formation dynamics and pressure $P_{d}$ at different $T_{d}$ was primarily studied for the normal PCF and the highbirefringence PCF[27, 28]. Following the previous work, this paper will provide a further analysis of the structure formation dynamics including hole position and hole centre shift in the drawing process.

\section{PCF structure and structure parameters}

\section{Basic structure parameters}

The cross section images of the PCF and its preform are shown in Fig. 1 (a) and (c) and the structural parameters of the PCF and its preform are described in Fig. 1 (b) and (d). The hole diameters of fibre and preform are defined as $2 r_{h, f}$ and $2 r_{h, p}$. The radii of fibre and preform are denoted as $r_{o, f}$ and $r_{o, p} . r_{f}$ is the distance between the centre of the fibre and that of the hole, and $r_{p}$ is the distance between the centre of the preform and that of the hole. The hexagonal rings from inner to outer are named as Ring 1, Ring 2, Ring 3, and Ring 4, respectively. Since some holes in Ring 4 are fully collapsed, only the holes in Ring 1, 2 and 3 will be considered and described in this work. Three red circled holes marked as $\mathrm{H}_{1}, \mathrm{H}_{2}$ and $\mathrm{H}_{3}$ at Ring 1, 2 and 3 in Fig. 1 (b) and (d) are typically selected to represent the structure formation of the holes in each ring. 
(a)

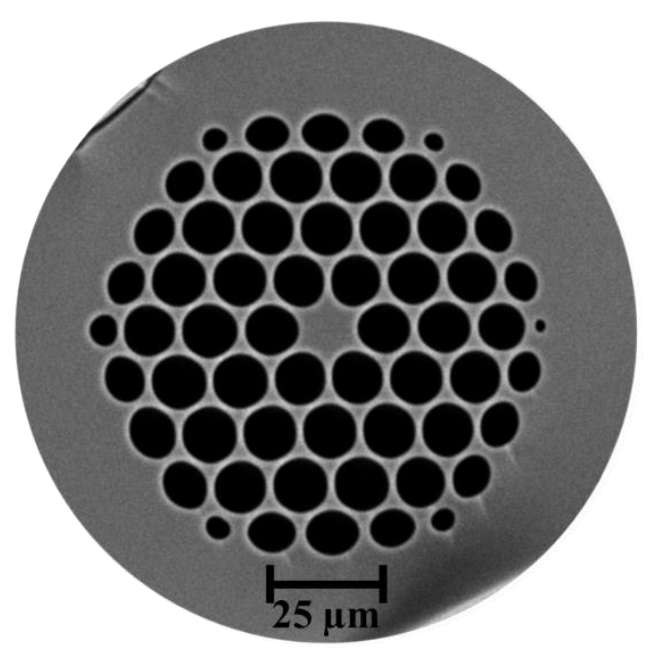

(c)

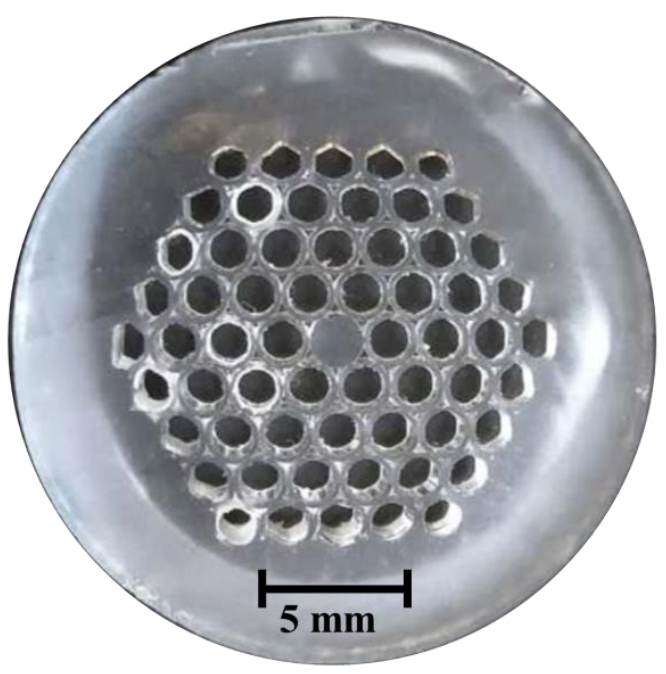

(b)

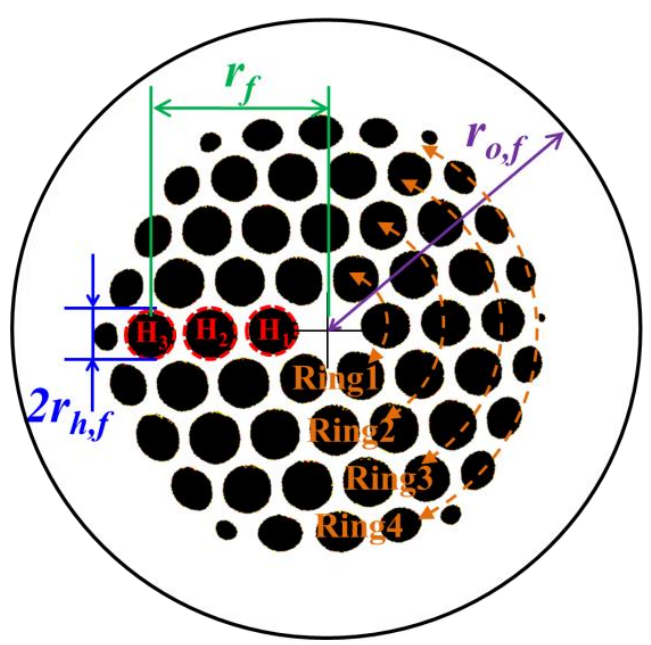

(d)

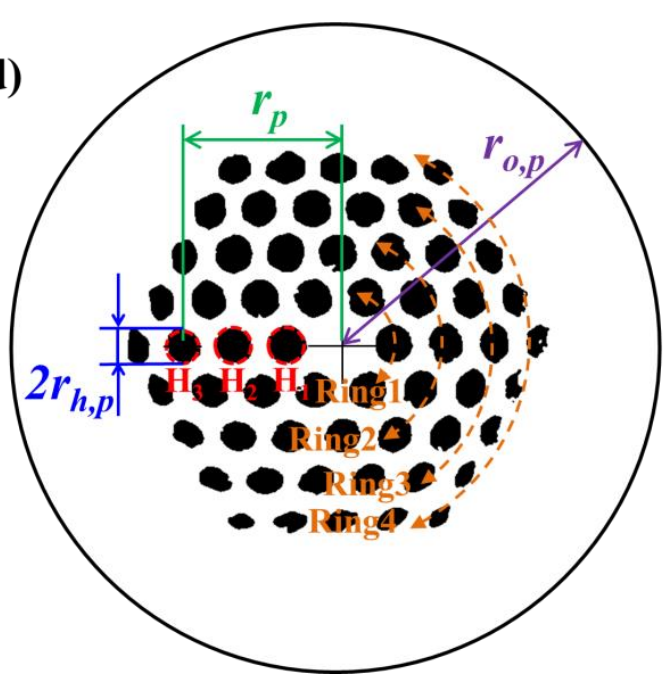

Fig. 1. Cross section images of a fabricated PCF (a) and its preform (c). The basic structural parameters are labeled in (b) for the PCF and (d) for the preform. The colors of (b) and (d) are calibrated for clearer views.

\section{Scale factor of hole-outer diameter ratio of fibre to preform $\boldsymbol{R}$}

For describing the collapse degree of the air hole, a scale factor $R$ is defined as:

$$
R=\frac{2 r_{h, f} / 2 r_{o, f}}{2 r_{h, p} / 2 r_{o, p}}=\frac{r_{h, f} / r_{o, f}}{r_{h, p} / r_{o, p}},
$$

where numerator term represents the hole-outer diameter ratio of fibre, and denominator term is the hole-outer diameter ratio of preform. According to the definition in Eq. (1), the change of air holes in PCF has the following four cases:

when $R=0$, holes are fully collapsed and $r_{h, f}=0$;

when $R<1$, holes are partially collapsed;

when $R>1$, holes are expanded;

when $R=1$, holes keeps the same ratio as those in the preform without any collapse or expansion (internal air pressure is balanced with the surface tension and drawing shear force). Here, $P_{d}$ is defined as the optimal pressure, $P_{o}$.

\section{$\underline{\text { Relative position of the hole in fibre } \boldsymbol{R}_{f}}$}


To study the relationship between the structure formation and hole position, the relative position of the hole in fibre $R_{f}$ is defined in Eq. (2):

$$
R_{f}=\frac{r_{f}}{r_{o, f}}
$$

\section{Relative position of the hole in preform $\boldsymbol{R}_{p}$}

Similarly, the relative position of the hole in preform $\left(R_{p}\right)$ is defined in Eq. (3):

$$
R_{p}=\frac{r_{p}}{r_{o, p}},
$$

\section{PCF fabrication results and discussion}

The PCF preform used was assembled by stacking a series of capillaries forming the hexagon shape with four rings and jacketing these capillaries with a silica tube. The inner diameter $D_{\text {inner }}$ and outer diameter $D_{\text {tube }}$ of the jacket tube are $18.93 \mathrm{~mm}$ and $24.97 \mathrm{~mm}$, and the ratio of outer to inner diameter of the capillary is 1.32 . The space between the capillaries and jacket tube was filled with solid rods for two reasons: 1. stabilizing the stacked hexagon PCF structure; 2. avoiding an excessive collapse of the holes at Ring 4 caused by high temperature at outer layer in the drawing process [1]. Then the stacked preform was fused on a lathe to relax the tolerance in drawing process by removing the interstitial area so that only lattice structure was concerned. The outer diameter of the fused preform $D_{\text {preform }}$ is $21.5 \mathrm{~mm}$ which means $25.8 \%$ of preform was collapsed (the area of cross section). Finally, the fused preform was drawn into fibres with furnace temperature $T_{d}=1860^{\circ} \mathrm{C}$ and $T_{d}$ $=1870^{\circ} \mathrm{C}$, drawing pressure $P_{d}=2.8 \sim 18.5 \mathrm{mbar}$, feeding rate $V_{f}=0.5 \mathrm{~mm} / \mathrm{min}$, and drawing rate $V_{d}=15$ $\mathrm{m} / \mathrm{min}$

\section{$\underline{\text { Scale factor } R \text { vs. drawing pressure } P_{d} \text { at different furnace temperature } T_{d}}$}

In our previous work [27], the relationship between drawing pressure $P_{d}$ and scale factor $R$ was discussed according to the cross section image scanned by the electron microscope shown in Fig. 2(a) and (b). Seen from Fig. 2(a), when $P_{d}=2.8$ mbar, the $R$ of $\mathrm{H}_{1}, \mathrm{H}_{2}$ and $\mathrm{H}_{3}$ are measured to be $0.43,0.36$ and 0.29 at $T_{d}=1860^{\circ} \mathrm{C}$. These less than one $\mathrm{R}$ values indicate the collapse of the holes. With the rise of $P_{d}$ to 9 mbar, the $R$ of each hole grows close to 1 , which means a balanced pressure is nearly provided at the boundary between the air hole and the glass. Finally, when $P_{d}$ is changed up to $12 \mathrm{mbar}$, the $R$ of each hole surpasses 1 . In this case, the holes are supposed to be expanded according to the definition of $R$. Therefore, the rule of $R$ vs. $P_{d}$ can be concluded as: 1 . when $P_{d}$ is set in a low region, the scale factor $R$ moves towards zero due to insufficient drawing pressure to resist the collapse tension; 2. when increasing the $P_{d}$, the high drawing pressure will overcome the collapse tension and blow up the hole, resulting in the increase of $R$.
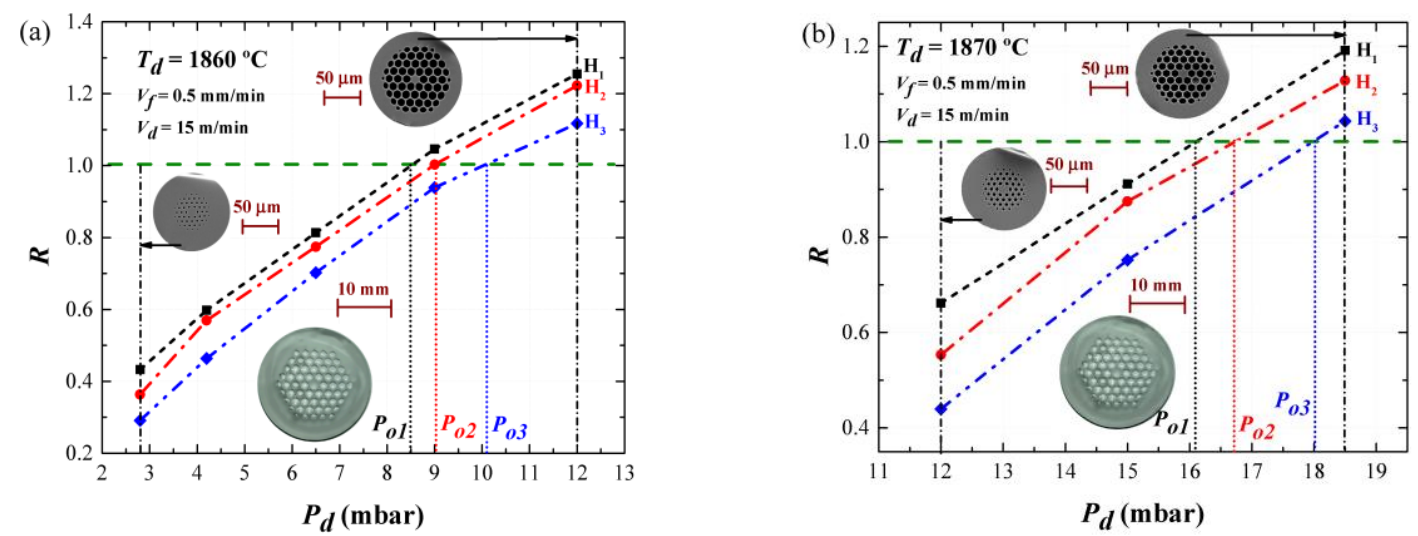

Fig. 2. The dependences of the scale factor $R$ on the drawing pressure $P_{d}$ for holes $\mathrm{H}_{1}, \mathrm{H}_{2}$ and $\mathrm{H}_{3}$. The results are shown for two furnace temperatures: $1860^{\circ} \mathrm{C}$ (a) and $1870^{\circ} \mathrm{C} \mathrm{(b)}$. Cross-section images of the preform and four PCFs with different drawing conditions are illustrated. The green dash lines represent $R=1$. The optimal pressures of holes $\mathrm{H}_{1}, \mathrm{H}_{2}$ and $\mathrm{H}_{3}$ are marked as $P_{o 1}, P_{o 2}, P_{o 3}$.

In addition, it has been found that the optimal pressure $P_{o}$ increases with the radial distance from the centre, which is $\mathrm{H}_{1}(8.5 \mathrm{mbar})<\mathrm{H}_{2}(9.1 \mathrm{mbar})<\mathrm{H}_{3}(10.2 \mathrm{mbar})$. Normally lower viscosity under higher $T_{d}$ leads to 
more collapse. As a result of a thermal gradient, a hole in the outer ring has a significantly higher temperature environment and a lower viscosity than the one in the inner ring. So that, as the experimental results show, a hole in the outer ring requires considerably increased $P_{d}$ to maintain the structure. Besides, a strong temperature dependence of $P_{o}$ is confirmed by comparing the optimal pressure $P_{o}$ in Fig. 2(a) and (b): when the furnace temperature $T_{d}$ increased by $10^{\circ} \mathrm{C}$, the $P_{o}$ of each hole increases by the rate of $1.77 \sim 1.89$ [27].

\section{$\underline{\text { Relative position of hole in fibre } R_{f} \text { vs. drawing pressure } P_{d} \text { at different furnace temperature } T_{d}}$}

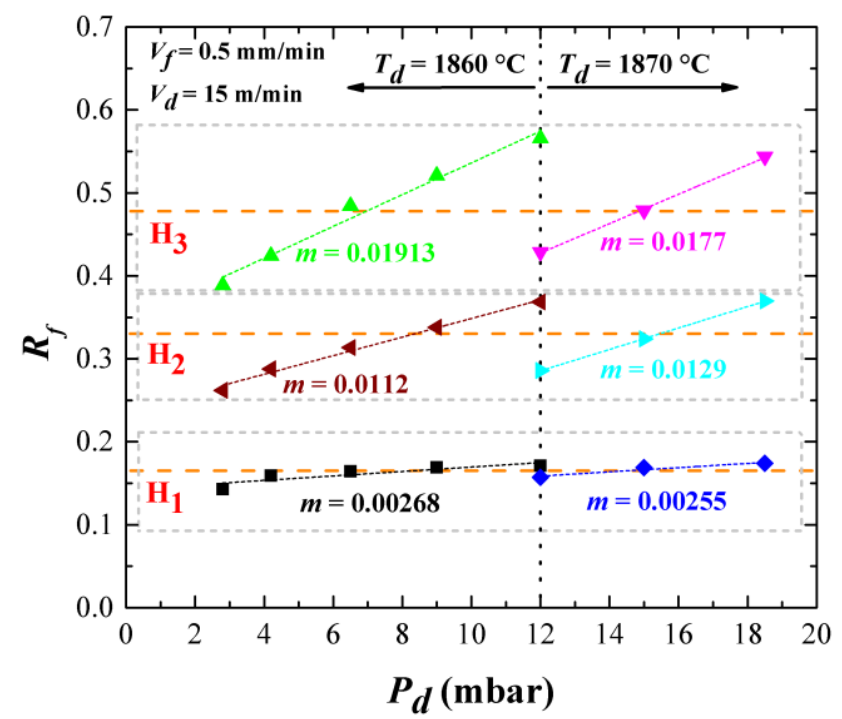

Fig. 3. The dependences of relative position of the hole in PCF $\left(R_{f}\right)$ upon the drawing pressure $P_{d}$ for holes $\mathrm{H}_{1}, \mathrm{H}_{2}$ and $\mathrm{H}_{3}$ at different temperature: $1860{ }^{\circ} \mathrm{C}$ (left part to the dotted line) and $1870{ }^{\circ} \mathrm{C}$ (right part to the dotted line). The orange dash lines represent the relative position of holes in the preform $\left(R_{p}\right) . m$ is the slope of $R_{f}$ to $P_{d}$.

The optimum PCF drawing is to achieve the targeted structure directly scaled down from its preform. However, the material migration always exists in PCF drawing due to the un-balance between the drawing pressure and the collapse tension. It will not only change the hole dimension, but also the position of hole in PCF regarding with that in the preform. The variation of the position of hole in PCF regarding with that in preform means that the movement of a hole in PCF is not in the proposition to the position of the hole in preform. In order to have a better control of the position of the holes in the PCF drawing process, the relative position of hole in fibre $\left(R_{f}\right)$ is introduced as Eq. (2) and its dependences on the drawing pressure and furnace temperature is further studied.

In Fig. 3, the $R_{f}$ of three tracked holes as a function of the drawing pressure $P_{d}$ of $2.8 \sim 18.5$ mbar at different furnace temperature $T_{d}$ are plotted. The $R_{f}$ of all three holes is almost increased (i.e. moving away from the center) with the increase of $P_{d}$. The $\mathrm{H}_{3}$ hole has the most significant movement with the $P_{d}$ because it has the largest slope $m$ of $R_{f}$ to $P_{d}$. Compared with the relative position of hole in preform $R_{p}$ (indicated as orange dash lines), the movement of the hole in the inner layer is less. Such phenomena might be attributed to two reasons: 1 . the effect of hole $\mathrm{H}_{3}$ located in the outer ring includes the additive effect from the inner rings; 2 . the holes in the outer ring have less restraint than those in the inner rings. For the latter reason, the hole $\mathrm{H}_{1}$ needs to push hole $\mathrm{H}_{2}$ and $\mathrm{H}_{3}$ if it tends to move outward, meanwhile, it is restricted by the high material viscosity in the centre of the PCF when it is going to move towards the centre of the fibre.

When the $P_{d}$ is increased to $9 \mathrm{mbar}$ at $T_{d}=1860^{\circ} \mathrm{C}$, an optimal condition is obtained for the hole $\mathrm{H}_{2}$, because the $R_{f}$ of hole $\mathrm{H}_{2}$ is 0.338 , which is only 0.005 larger than the relative position in preform $R_{p}$. In the meantime, the difference between the $R_{f}(0.169)$ and the $R_{p}(0.167)$ of $\mathrm{H}_{1}$ is very small at this drawing condition as well. When the temperature is increased by $10^{\circ} \mathrm{C}$, the $R_{f}$ is found to be lower than that at $1860^{\circ} \mathrm{C}$ with the same $P_{d}=12 \mathrm{mbar}$. Considering that the optical property in PCF is largely decided by the inner layers [29], the optimal drawing condition at $1870^{\circ} \mathrm{C}$ is suggested to be 15 mbar according to the $R_{f}$ of $\mathrm{H}_{1}$ and $\mathrm{H}_{2}$. Hence, drawing conditions of 9 mbar at $1860^{\circ} \mathrm{C}$ and $15 \mathrm{mbar}$ at $1870^{\circ} \mathrm{C}$ are favorable settings for maintaining the hole positions in preform after PCF drawing.

\section{$\underline{\text { Scale factor } \boldsymbol{R} \text { vs. relative position of hole in fibre } \boldsymbol{R}_{f}}$}

As discussed above, both of the scale factor $R$ and relative position of hole in fibre $R_{f}$ can be significantly affected by the drawing pressure and furnace temperature. Thereby, it is meaningful to investigate how these two parameters react to the drawing condition correspondingly and search for a drawing condition to optimize $R$ 
and $R_{f}$ simultaneously. In Fig. 4 , the relationship of $R$ and $R_{f}$ calculated for $\mathrm{H}_{1}, \mathrm{H}_{2}$ and $\mathrm{H}_{3}$ are plotted with different drawing conditions.

Under the same drawing pressure $P_{d}$ and furnace temperature $T_{d}$, the $R$ drops with increasing $R_{f}$, which means the hole at inner layer has less collapse $(0<R<1)$ or more expansion $(R>1)$ than that at outer layer. Such correlation between the $R$ and $R_{f}$ holds for any investigated drawing condition shown in Fig. 4 (a) and (b). Knowing that the optimal $R$ is 1 and the optimal $R_{f}$ equals to the relative position of hole in the preform $R_{p}$, the optimal $R$ and $R_{f}$ are plotted by dash lines accordingly. Thus, it is easy to understand that three intersections are the optimal points for three holes in the Fig. 4 (a) and (b). Therefore, an optimal drawing condition is $P_{d}=9$ mbar and $T_{d}=1860^{\circ} \mathrm{C}$, where all $R$ values of three holes have the smallest difference with each other, and the $R_{f}$ of $\mathrm{H}_{1}$ and $\mathrm{H}_{2}$ locate close to their best position.

In an ideal situation, the $R$ and $R_{f}$ should fulfill all optimal conditions. However, the scale factor $R$ has more or less dependence on the $R_{f}$, indicating that it is difficult to make all of the $R$ in each ring equal to one at the same time. Hence, one strategy to solve this problem is to reduce the dependence of $R$ on $R_{f}$. In Fig. 5, the slope $s$ of the $R$ to the $R_{f}$ with same drawing condition is calculated to represent the degree of the dependence of the $R$ on the $R_{f}$. At both $T_{d}$, slope $s$ tends to decrease with increasing $P_{d}$ and is likely to converge to some range $(0.3 \sim 0.35$ at $1860{ }^{\circ} \mathrm{C}$ ). This result implies that the strength of the dependence of $R$ on $R_{f}$ can be reduced by increasing the $P_{d}$. In other words, the holes at each ring will have a similar deformation behavior under high drawing pressure. Moreover, by decreasing the temperature from $1870^{\circ} \mathrm{C}$ to $1860^{\circ} \mathrm{C}$, the $s$ drops significantly from 0.82 to 0.35 with drawing pressure of 12 mbar showing a great dependence of $s$ on the furnace temperature. Based on these analyses, one can have a low $R$ dependence on $R_{f}$ by carefully lowering the furnace temperature and obtain an optimal $R$ by adjusting the $P_{d}$ afterward. However, attention on the corresponding variation of $R_{f}$ on the $P_{d}$ needs to be paid as well.
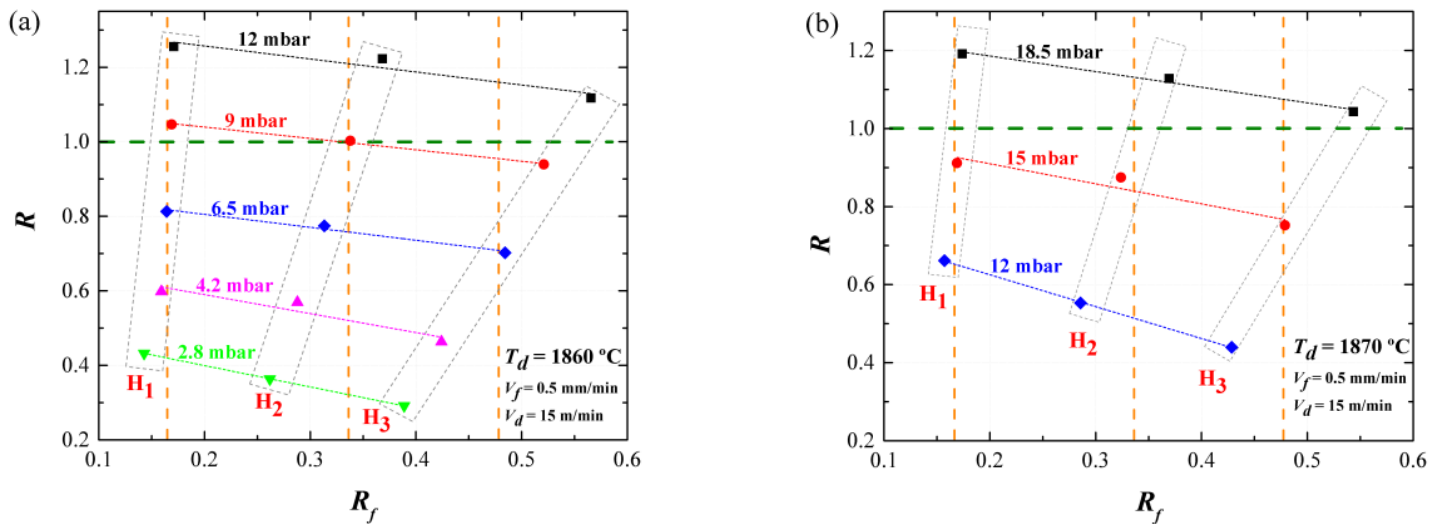

Fig. 4. The dependence of scale factor $R$ on the relative position of hole in the fibre $R_{f}$ for holes $\mathrm{H}_{1}, \mathrm{H}_{2}$ and $\mathrm{H}_{3}$ at $T_{d}=$ $1860^{\circ} \mathrm{C}$ (a) and $T_{d}=1870^{\circ} \mathrm{C}$ (b). The orange dash lines represent the relative position of hole in the preform $R_{p}$. The green dash lines represent $\mathrm{R}=1$.

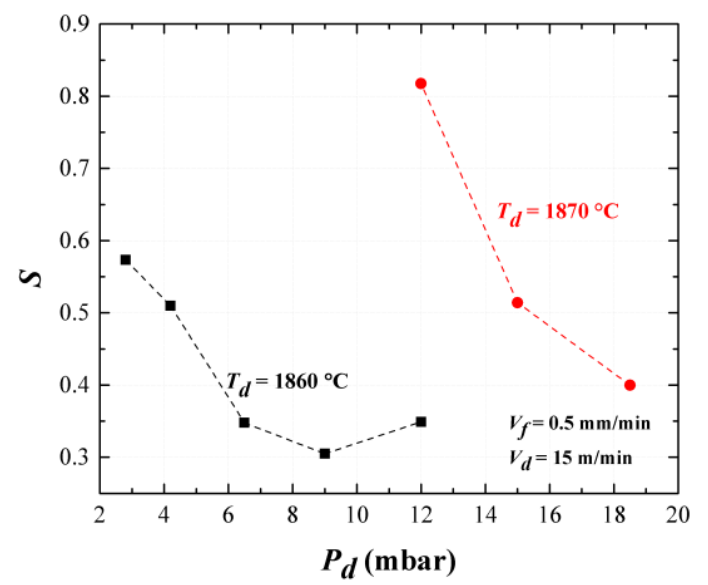

Fig. 5. The dependence of the slope $s$ of the scale factor $R$ to the relative position of hole in the fibre $R_{f}$ upon the drawing pressure $P_{d}$ for holes $\mathrm{H}_{1}, \mathrm{H}_{2}$ and $\mathrm{H}_{3}$ at different furnace temperatures. 


\section{Conclusion}

Optical features of PCFs are determined by the air hole structure, formed by its preform design and the following control of the conditions in PCF drawing process. Two new structure parameters-scale factor $R$ and relative position of hole in fibre $R_{f}$ have been introduced to assess the structure formation compared with its preform. The relationships between scale factor $R$, relative position of hole in fibre $R_{f}$ and drawing conditions, including drawing pressure $P_{d}$ and furnace temperature $T_{d}$, have further been investigated by a series of PCFs drawn under different drawing conditions. To maintain the PCF structure, the optimal pressure is required, which shows a strong temperature dependence, while to maintain the hole position of PCF as the preform, two sets of the drawing conditions are favorable. This investigation predicts that there exists an optimal condition to achieve perfect PCF structure without hole collapse/expansion as well as hole shift as the design.

Acknowledgment: We thank AFRL, HEL-JTO and AFOSR/AOARD for providing the funding for this work under grant number FA2386-16-1-4031. T.K. thanks the support of the European Community Pacific Atlantic Network for Technical Higher Education and Research project.

\section{References}

[1] Buczynski R. Photonic crystal fibers. Acta Phys. Pol. Series A, 2004, 106(2): 141-167.

[2] Birks T A, Knight J C, Russell P S J. Endlessly single-mode photonic crystal fiber. Opt. Lett., 1997, 22(13): 961.

[3] Stone J M. Photonic crystal fibres and their applications in the nonlinear regime. PhD Thesis. Bath: University of Bath, 2009.

[4] Michie A, Canning J, Lyytikäinen K, Aslund M, Digweed J. Temperature independent highly birefringent photonic crystal fibre. Opt. Express, 2004, 12(21): 5160-5165.

[5] Wadsworth W J, Percival R M, Bouwmans G, Knight J C, Russell P S J. High power air-clad photonic crystal fibre laser. Optics Express, 2003, 11(1): 48-53.

[6] Cook K, Canning J, Holdsworth J, Dewhurst C. Stable CW single-mode photonic crystal fiber DFB ring laser. Journal of Electronic Science and Technology of China, 2008, 6(4): 442-444.

[7] Groothoff N, Canning J, Ryan T, Digweed, K, Inglis H. Distributed feedback photonic crystal fibre (DFB-PCF) laser. Optics Express, 2005, 13(8): 2924-2930.

[8] Chen T, Chen R, Jewart C, Zhang B, Cook K, Canning J, Chen K. Regenerated gratings in air-hole microstructured fibers for high-temperature pressure sensing. Optics Letters, 2011, 36(18): 3542-3544.

[9] Michie A, Canning J, Bassett I, Haywood J, Digweed K, Ashton B, Stevenson M, Digweed J, Lau A, Scandurra D. Spun elliptically birefringent photonic crystal fibre for current sensing. Measurement Science and Technology, 2007, 18(10): 3070-3074.

[10] Cook, K, Canning J, Holdsworth J. Birefringent bragg gratings in highly-nonlinear photonic crystal fiber. Journal of Electronic Science and Technology of China, 2008, 6(4): 426-428.

[11] Lyytikäinen K. Numerical simulation of a specialty optical fibre drawing process. In: $27^{\text {th }}$ Australian Conference on Optical Fibre Technology. Australia: Sydney, 2002, 134-136.

[12] Lyytikäinen K, Råback P, Ruokolainen J. Numerical simulation of a specialty optical fibre drawing process. In: $4^{\text {th }}$ Int. ASME/JSME/KSME Symp. Computational Technologies for Fluid/Thermal/Chemical/Stress System With Industrial Applications. Canada: Vancouver, BC, 2002, $267-275$.

[13] Wynne R M. A Fabrication process for microstructured optical fibers. J. Lightwave Technol., 2006, 24(11): 4304-4313.

[14] Fitt A D, Furusawa K, Monro T M, Please C P, Richardson D J. The mathematical modelling of capillary drawing for holey fibre manufacture. J. Eng. Math., 2002, 43(2-4): 201-227.

[15] Fitt A D, Furusawa K, Monro T M, Please C P. Modeling the fabrication of hollow fibers: capillary drawing. J. Lightwave Technol., 2001, 19(12): 1924-1931.

[16] Yarin A L, Gospodinov P, Roussinov V I. Stability loss and sensitivity in hollow fiber drawing. Physics of Fluids, 1994, 6(4): 1454-1463.

[17] Gospodinov P, Yarinz A L. Draw resonance of optical microcapillaries in non-isothermal drawing. Int. 
J. Multiphase Flow, 1997, 23(5): 967-976.

[18] Wadsworth W, Witkowska A, Leon-Saval S, Birks T. Hole inflation and tapering of stock photonic crystal fibres. Opt. Express, 2005, 13(17): 6541-6549.

[19] Chen Y, Birks T A. Predicting hole sizes after fibre drawing without knowing the viscosity. Opt. Mater. Express, 2013, 3(3): 346-356.

[20] Lyytikäinen K, Zagari J, Barton G, Canning J. Heat transfer in a microstructured optical fibre preform. In: $11^{\text {th }}$ International Plastic Optical Fibers Conference. Japan: Tokyo, 2002, 53-56.

[21] Lyytikäinen K, Zagari J, Barton G, Canning J. Heat transfer within a microstructured polymer optical fibre preform. Modelling and Simulation in Materials Science and Engineering, 2004, 12(3)ss: S255.

[22] Lyytikainen K, Canning J, Digweed J, Zagari J. Geometry control of air-silica structured optical fibres using pressurisation. In: Microwave and Optoeletronics Conference. Brazil: Parana, 2003, 1001-1005. Proceedings of the 2003 SBMO/IEEE MTT-S Interational, IEEE, 2003, 2:1001-1005.

[23] Lyytikäinen K J. Control of complex structural geometry in optical fibre drawing. PhD Thesis. Sydney: University of Sydney, 2004,157-176.

[24] Chen Y. Hole control in photonic crystal fibres. PhD Thesis. Bath: University of Bath, 2013, 52-61.

[25] Guo T Y, Luo S Q, Li H L, Jian S L. Control of the fabrication parameters during the fabriation of photonic crystal fibers. Acta Phys., 2009, 58(9): 6308-6315.

[26] Lyytikainen K, Canning J, Digweed J, Zagari J. Geometry control of air-silica structured optical fibres. In: Optical Internet \& Australian Conference on Optical Fibre Technology. Australia: Melbourne, 2003, $137-140$

[27] Wang W, Karpisz T, Tafti G, Canning J, Cook K, Luo Y, Peng G. Optimal pressure for tuning the lattice structure of photoinic crystal fibre. In: $3^{\text {rd }}$ the Australian and New Zealand Conference on Optics and Photonics. New Zealand: Queenstown, 2017, Paper 141.

[28] Tafti G, Wang W, Karpisz T, Canning J, Cook K, Luo Y, Wang S, Peng G. Fabrication and structure of H-Bi Micro-structured optical fibres. In: $3^{\text {rd }}$ the Australian and New Zealand Conference on Optics and Photonics. New Zealand: Queenstown, 2017, Paper 142.

[29] Hoo Y L, Jin W, Shi C, Ho H L., Wang D N, Ruan S C. Design and modeling of a photonic crystal fiber gas sensor. J. Applied Optics, 2003, 42(18): 3509-3515.

E-mail: wenyu.wang@student.unsw.edu.au, g.peng@unsw.edu.au 